\title{
Control of nonlinear collapse in magnetooptical Kerr media
}

\author{
Katarzyna A. Rutkowska, ${ }^{* 1,2}$ Yoav Linzon, ${ }^{2}$ Boris A. Malomed, ${ }^{3}$ and Roberto Morandotti ${ }^{2}$ \\ ${ }^{1}$ Faculty of Physics, Warsaw University of Technology, 00-662 Warsaw, POLAND, \\ ${ }^{2}$ INRS-EMT, University of Québec, Varennes, J3X 1S2 Québec, CANADA \\ ${ }^{3}$ Department of Physical Electronics, Faculty of Engineering, Tel Aviv University, Tel Aviv 69978, ISRAEL
}

Received October 28, 2009; accepted December 29, 2009; published December 31, 2009

\begin{abstract}
We demonstrate a novel approach to the control of the collapse of optical beams in Kerr media. To this end, we consider a combination of two magnetooptical (MO) phenomena, the CottonMouton and Faraday effects, in order to introduce a suitable and tunable interplay between the induced linear and circular birefringences. Our numerical analysis confirms that a properly applied external magnetic field may alter the dynamics of the beam propagation in magnetic selffocusing bulk Kerr media. For a chosen value of the optical power, the acceleration, the delay or even the arrest of the collapse can be achieved. We demonstrate experimentally the magnetically-induced acceleration of the collapse in a ferrimagnetic yttrium iron garnet crystal (YIG).
\end{abstract}

High-power light propagation in multidimensional selffocusing Kerr media is generally unstable, leading, in particular, to wave collapse [1]-[2]. Such collapse occurs when the input optical power exceeds the critical (threshold) value, which may lead to significant changes in the spatial beam profile (e.g., filamentation), as well as to material damage. Collapsing optical beams in Kerr media have been thoroughly investigated. One of the challenges facing this field of interest is to control and arrest the collapse. The latter is essential to prevent the collapse-induced material damage, as well as to stabilize the propagation of spatial solitons in bulk nonlinear media. So far, different mechanisms for the management of the collapse have been theoretically explored, by applying suitable modifications to the conventional form of the nonlinear Schrödinger equation (NLSE) [1]-[5]. Additional physical effects (e.g. resulting from the higher-order interactions between light and matter), such as multiphoton absorption, plasma formation, temporal dispersion, energy dissipation and nonparaxial corrections, have been considered [3]-[5]. These effects are taken into account by introducing new terms in the NLSE. It has been demonstrated that such a modified equation (the generalized NLSE) may possess solutions in the form of solitons (more precisely, solitary waves), under certain conditions imposed onto the beam and the medium. In particular, it has been shown that for nonlocal or saturating nonlinearities the collapse may be suppressed, allowing for the formation of spatial solitons in bulk media [6].

*E-mail: kasia@if.pw.edu.pl
In this work we demonstrate that optical birefringence can also act as a powerful tool for the control of the optical collapse. We show that the power and the phase transfer between the beam polarization components add suitable terms to the standard system of coupled NLSEs, allowing for the collapse control. Specifically, in the case of magnetic nonlinear media, we propose to take advantage of the superposition [7] of the linear $\left(\Delta n_{l}\right)$ and circular $\left(\Delta n_{c}\right)$ birefringences, induced in the presence of an external magnetic field via the Cotton-Mouton and Faraday effects, respectively [7]-[8]. We demonstrate that, for a fixed value of the input optical power (exceeding the critical one), the application of an appropriate magnetic field (i.e., depending on its strength and direction) may change the beam-propagation dynamics in such a way that acceleration, delay or even complete elimination of the collapse may occur [9].

Our theoretical model is based on a system of coupled $(2+1) D$ scalar modified NLSEs describing the evolution of the complex electric field amplitudes of the two polarizations in the presence of the self-focusing Kerr nonlinearity. Assuming the circular symmetry of the problem and applying typical normalization factors [1], the propagation equations can be written in the scaled form,

$$
\begin{array}{r}
i \frac{\partial U_{R, L}}{\partial \widetilde{z}}+\frac{1}{2} \widetilde{\nabla}_{\perp}^{2} U_{R, L}+\left(\left|U_{R, L}\right|^{2}+2\left|U_{L, R}\right|^{2}\right) U_{R, L}+ \\
\pm b U_{R, L}+c U_{L, R}=0
\end{array}
$$

where: $U_{R, L}$ is the normalized slowly varying envelope for left- and right- circularly polarized components, $\widetilde{\nabla}_{\perp}^{2}=\frac{\partial^{2}}{\partial \widetilde{r}^{2}}+\frac{1}{\widetilde{r}} \frac{\partial}{\partial \widetilde{r}}$ is the transverse Laplacian, $\tilde{r}=r / w_{0}$ and $\tilde{z}=z /\left(2 L_{D}\right)$ are the normalized transverse and longitudinal coordinates, respectively, $w_{0}$ is the initial width of the beam, and $L_{D}$ is the diffraction length. Two additional terms describe the magnetically-induced birefringences. They are represented by the real coefficients $b$ and $c$, which account for the circular and linear birefringences, respectively. More precisely, $c$ and $b$ describe the CottonMouton and Faraday effects in MO media, and are strongly related to the changes in the refractive index of the linearly and circularly polarized waves (i.e., $b \sim \Delta n_{c}$ and $c \sim \Delta n_{l}$ ). While Eqs. (1) give rise to collapsing 
solutions, we have performed a detailed numerical analysis to determine if the additional terms, which depend on the value and direction of the external magnetic field, may stabilize or destabilize the beams against the collapse, for the certain geometry and initial conditions. The results of the simulations are shown in Fig. 1. In the absence of the magnetic field (i.e., when no magnetically-controlled birefringence is induced), wellknown dynamics occurs. For low input powers the beam diffracts (Fig. 1(a)). Its spatial divergence is reduced when the optical power is increased. Eventually, when the power exceeds the critical value, the collapse takes place, after a finite propagation distance (Fig. 1(b)). The collapse distance is reduced with the further increase of the optical power. For a fixed value of the input power, the collapse distance can be modified by taking advantage of the magnetically-controlled birefringences, whose strengths are described by the coefficients $b$ and $c$. The collapse may be accelerated (i.e., it takes place after a shorter propagation distance) by the linear birefringence alone, as shown in Fig. 1(c). When both the linear and circular birefringences are present, the collapse distance may be extended (see Fig. 1(d)), i.e., the beam may indeed be partly stabilized against the onset of the collapse.

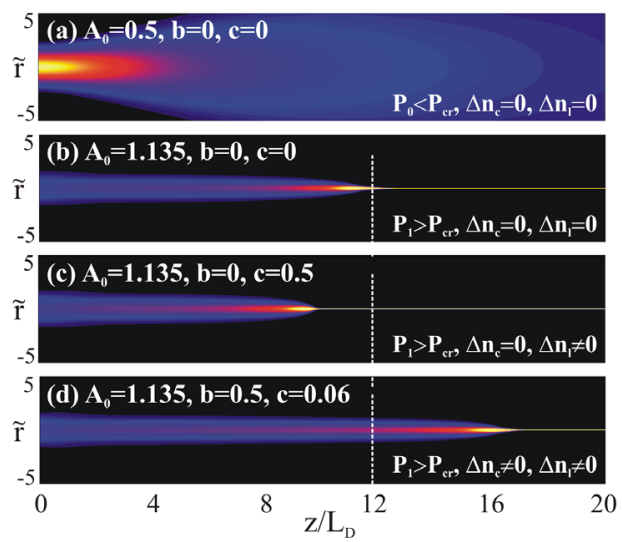

Fig.1. Results of numerical simulations of the beam-propagation dynamics. The latter depends on the input optical power, $P$ (here $P_{c r}$ is the critical value), and on the magnetically-induced linear $\left(\Delta n_{l}\right)$ and circular $\left(\Delta n_{c}\right)$ birefringences. $\mathrm{A}_{0}$ is the initial amplitude of the Gaussian beam.

The delay and, eventually, the arrest of the collapse (with respect to the situation with no external magnetic field, for the same optical power associated to the input beam) occurs only for a particular combination of the birefringence coefficients, resulting in a specific interplay between the amplitude and phase mixing of the beam polarization components. The range of the birefringence coefficients for which high-power beams do not collapse can be found in Fig. 2(a), depicting the output width of the beam after a propagation distance $z_{\max }=100 L_{D}$. If the collapse takes place at $z \leq z_{\max }$ the final width of the beam is set to zero, being represented by the grey area in Fig. 2(a), otherwise the actual beam width at $z=z_{\max }$ is shown in the graph. The simulations allow us to conclude that, at a fixed value of the optical power and for $c>b$, the collapse can be accelerated, whereas for $b>c$ the delay or even the suppression of the collapse is possible. In the latter case, the particular combination of the birefringence coefficients eventually leads to the diffraction of the beam, in spite of its high input power.

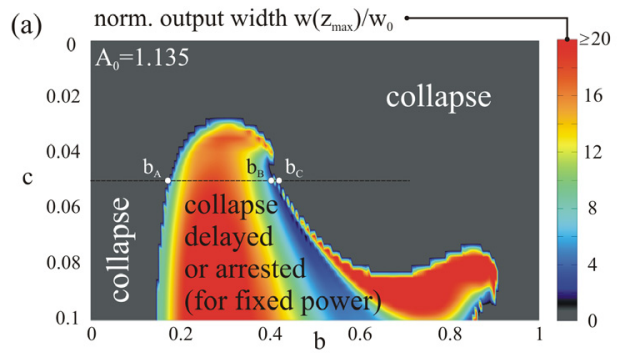

(b)
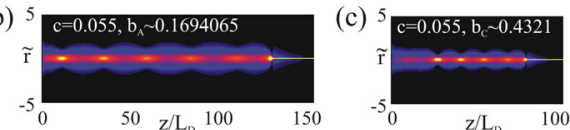

(d)

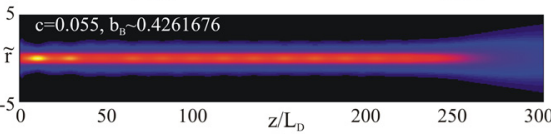

Fig.2. (a) The normalized width of the output beam, obtained for inputbeam amplitude $A_{0}=1.135$ and propagation distance $z_{\max }=100 L_{D}$, as a function of the magnetically-induced birefringences. (b)-(d) The beam propagation for three particular combinations of the birefringence coefficients, namely for $c=0.055$ and $b=b_{A}, b_{B}$, and $b_{C}$, respectively, as specified by the three points marked in (a).

The experimental configuration and material parameters for the available MO crystals impose limitations on the achievable range of birefringence coefficients. To date, only the acceleration of the collapse was observed in the experiment [9]. Nevertheless, we can theoretically extend the possible range of parameters to investigate different dynamical ranges in more general settings. For a fixed value of the linear birefringence coefficient $c$, three critical (characteristic) values of the linear birefringence coefficient $b$, labelled as $b_{i=A, B, C}$, exist, see Fig. 2(a). Two of them, $b_{A}$ and $b_{C}$, correspond to the transition between collapsing and diffracting solutions, defined as the parameters for which the collapse distance can be significantly extended (Fig. 2(b)(c)). The third critical value, $b_{B}$, refers to the regime for which the beam spatial divergence is reduced in such a way that the quasi-stable propagation is achieved before diffraction eventually takes place (Fig. 2(d)). In such a case, the critical power necessary for the onset of the collapse effectively increases. In general, there exist quasi-stable solutions that are characterized by the same input power but different values of the birefringence parameters, i.e., for a particular combination of coefficients $b$ and $c$, the quasi-stable propagation of the beam, before it eventually collapses or spreads out, may 
be extended for distances as long as several hundreds of the diffraction lengths. To conclude the presentation of the theoretical results, it is worth mentioning that very similar results have also been obtained in a semianalytical form by means of the variational approximation, which is a method widely used in nonlinear optics [10]. These results will be reported elsewhere.

Our experiments were performed in a bulk crystal of YIG, which is commonly used for the fabrication of commercial optical isolators [8]. In addition to its remarkable MO properties, this ferrimagnetic cubic dielectric crystal is highly transparent to near infrared radiation. Our measurements were made at a wavelength of $1.2 \mu \mathrm{m}$ (using an OPA laser system delivering 200-fs pulses at a repetition rate of $1 \mathrm{kHz}$ and with a peak power of up to $50 \mathrm{MW}$ ), which was found to provide the best trade-off between significant MO coefficients and low absorption losses in YIG.

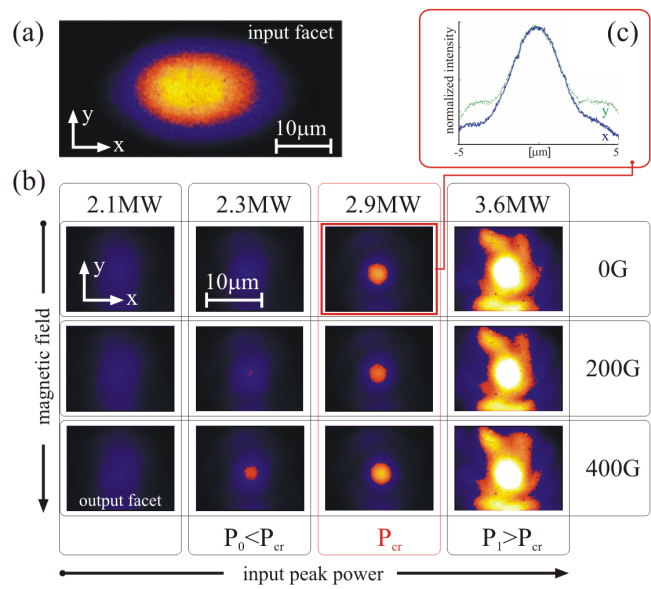

Fig.3. Experimental results. (a) The beam profile as imaged at the input facet of the YIG crystal. (b) Images of the beam profiles at the output facet as a function of input peak power $P$ and of the applied magnetic field. (c) Cross sections along the $\mathrm{x}$ and $y$ directions for the central spot of the beam collapsing at $2.9 \mathrm{MW}$, with no magnetic field applied.

The Cotton-Mouton geometry, where a beam propagates perpendicular to the direction of the magnetic field, was fixed in our experimental setup, corresponding to the condition $\Delta n_{l}>>\Delta n_{c}$, under which only the acceleration of the collapse may be observed, as predicted by the simulations and by the variational approximation. However, near the collapse point, the paraxial approximation ceases to hold, leading in turn to a non negligible contribution of the Faraday term.

Our experimental results are summarized in Fig. 3(b), where we show the beam profiles observed at the output facet of the crystal as a function of the input power and of the magnetic field. When no field is applied, a highpower spot appears at the center of the diffraction background at the critical power $P_{c r}=2.9 \mathrm{MW}$. This spot is circularly symmetric (even if the input beam profile was elliptic, as in Fig. 3(a)) and is thus fitting the so-called
Townes' profile [11] (see Fig. 3(c)). In turn, this is a strong indication of the onset of the collapse at the output facet of the crystal. At higher powers, the collapse takes place inside the crystal, and the filamentation of the beam is observed at the output facet. The application of the magnetic field to the YIG crystal changes the conditions for the collapse, and its onset at the output facet is observed at lower powers. The predicted decrease of the threshold (critical) power, observed when the magnetic field is increased, is thus demonstrated, with the strongest collapse-acceleration effect observed at $B=400 \mathrm{G}$ (Fig. 3(b)).

To the best of our knowledge, this is the first experimental demonstration of the nonlinear beamcollapse management in Kerr media where the birefringences can be tuned via the application of an external magnetic field. In order to observe the delay or arrest of the collapse, a change in the experimental configuration (a different direction of the light propagation with respect to the magnetic field), or the use of other MO materials are required, to fulfill the condition of a stronger contribution of the circular birefringence over its linear counterpart $\left(\Delta n_{c}>>\Delta n_{l}\right)$ in the presence of the magnetic field. These conditions can be readily implemented and the proposed technique (involving the combined effect of the magnetically-induced circular and linear birefringences) may be employed for the complete control of all-optical lensing mechanism in self-focusing media. In this context, we believe that our studies will help to open new possibilities for the development of magnetically assisted nonlinear optics.

K.A.R acknowledges a Marie Curie Fellowship (MOIF-CT-2006-039600). R.M. acknowledges support from an NSERC Strategic Grant, from O E Land LTD and from TeraXion LTD.

\section{References}

[1] R.W. Boyd, Nonlinear optics (San Diego, Academic Press 2008).

[2] C. Sulem, P.L. Sulem, The nonlinear Schrödinger equation: selffocusing and wave collapse (New York, Springer 1999).

[3] L. Bergé, Phys. Rep. 303, 259 (1998).

[4] Y.S. Kivshar, D.E. Pelinovsky, Phys. Rep. 331, 117 (2000).

[5] B.A. Malomed, D. Mihalache, F. Wise, and L. Torner, J. Optics B: Quant. Semicl. Opt. 7, R53 (2005).

[6] M. Peccianti, K.A. Brzdakiewicz, G. Assanto, Opt. Lett. 27, 1460 (2002); O. Bang, W. Krolikowski, J. Wyller, J.J. Rasmussen, Phys. Rev. E 66, 046619 (2002).

[7] R. Kurzynowski, W.A. Woźniak, Optik 115, 473 (2004).

[8] A.K. Zvezdin, V.A. Kotov, Modern Magnetooptics and Magnetooptical Materials (New York, Taylor and Francis 1997).

[9] Y. Linzon, K.A. Rutkowska, B.A. Malomed, R. Morandotti, Phys. Rev. Lett. 103, 053902 (2009).

[10] B.A. Malomed, in Progress in Optics 43, 71 (E. Wolf, North Holland, Amsterdam, 2002).

[11] R.Y. Chiao, E. Garmire, C.H. Townes, Phys. Rev. Lett. 13, 479 (1964). 